\title{
Control Design of Tunnel Robot Based on ARM
}

\author{
Xiaolong Zhou ${ }^{1}$, a, Wei Song ${ }^{1}$, Jiangcheng Wang ${ }^{1}$, Xinping $\mathrm{Li}^{2}$ \\ ${ }^{1}$ State Grid Chongqing Electric Power Co. Electric Power Research Institute, 401123, Chongqing, \\ China
}

2The Smartech Institute, 518057, Shenzhen, China

a270642662@qq.com

Keywords: Tunnel robot, ARM, WIFI, autonomous obstacle avoidance

\begin{abstract}
In order to improve tunnel in long, narrow tunnel environment flexible robot control and autonomous obstacle avoidance ability, designed the control platform based on ARM, more modular sensor fusion, through WIFI communications and figure and raspberry pie auxiliary control system in the field of image processing, signal processing, robot applied to the tunnel. The control system design, has solved the tunnel under complicated environment communication impeded, the figure is not clear, cannot independent walls and other control problems, at the same time solve the operate of people can be far away from the tunnel environment, the problem of inspection. Robot can be realized for traditional cable tunnel without modification inspection provides the control scheme.
\end{abstract}

\section{Introduction}

In recent years, with the increase of tunnel engineering, tunnel radio communication has been seriously; domestic and foreign scholars have done a lot of research, some theories or utility have been proposed, such as "a rectangular tunnel wireless communication model" tent law based on the "influence of polarized antenna for wireless communication in the tunnel," a comprehensive use transmitter and receiver antennas diversity technique "etc, but they are used for the traffic tunnel environment, the cable and the directional narrow tunnel is not very ideal

WIFI is a wireless technology by which personal computer, handheld devices (such as pad, mobile phone and other terminal) connected to each other. in fact, it is a high frequency radio signal. Thanks to the rapid development of WIFI technology in recent years, although the WIFI is a short distance wireless technology, but with high power and high power router, antenna and multi AP network structure, it is possible to build WIFI network in a tunnel. At present, this technology has been used in hotels, hotels and other places, and the technology is mature.

Building WIFI net in the tunnel environment to transmit data and images, and then using the raspberry pie to analyse image signal and control signal; the use of ARM platform for multi sensor signal processing and controlling the robot is more practical at present.

\section{Design of mechanical structure}

\subsection{Structural design}

The tunnel is divided into two parts: mobile robot chassis and yuntai. The mobile chassis include a vehicle body, walking track, front swing arm structure for robot cruise, obstacle avoidance, flexible movement; head including a telescopic arm and 2 DOF camera structure, is used for line inspection, condition monitoring, nameplate sign recognition, as shown in figure 1.

\subsection{Mobile chassis}

In the mechanism, crawler chassis is the core component. It has the following functions: walking, climbing, climbing, turning and stepping over the threshold and obstacle avoidance functions. The mobile chassis is mainly composed of a body, a swing arm motor, walking motor, walking track, front swing arm etc. Walking motor and swing arm motor with sealed water shield, ensure the wading depth of $200-300 \mathrm{~mm}$, involving the depth of mud $50-100 \mathrm{~mm}$. With a walking motor driven walking crawler unilateral pendulum and arm crawler, transfer the output to the front through the chain track 
driving wheel, the swing arm driving wheel and crawler driving wheel concentric overlap, realize the synchronous rotation of unilateral track. the swing arm with a dual output worm drive gear motor, swing arm shaft through the swing arm driving wheel and crawler driving wheel hollow, transferred to the swing arm in the obstacle can be achieved when the swing arm and lifting, so as to realize the synchronization and simplified control, as shown in figure 2.

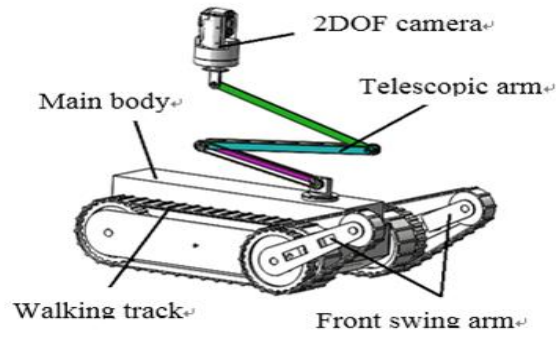

Figure 1 Structure of robot.

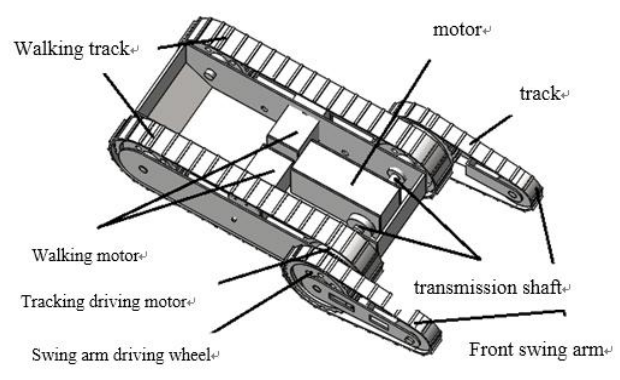

Figure 2. Move chassis.

\subsection{Head structure}

Head structure is a three arm linkage folding lifting platform with 7 degrees of freedom, as shown in figure 3. The most popular in the market at present is the folding arm and a telescopic arm. The following is the comparison of folding arm and telescopic arm:

(1) Folding arm fast, high efficiency; the telescopic arm mostly uses wire rope retracting mechanism, but the working efficiency is relatively low.

(2) Three degrees of freedom head as well, the folding arm can expand the scope of work, but only lift in the vertical direction.

(3) In operation, the folding arm and the telescopic arm of linkage is the same, both can lift by simple operation.

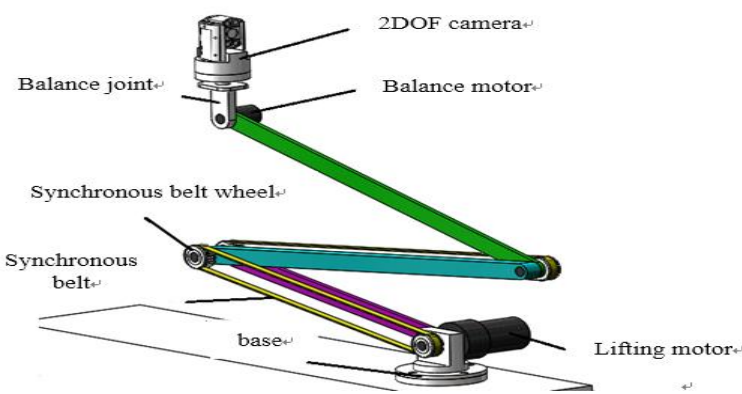

Figure 3. The structure of Yuntai.

The head movements using section three folding arm, a motor drive, two synchronous belt transmission, the realization of the three arms and the folding and unfolding. This can reduce the number of motors and decrease control difficulty. At the same time, there is a joint installed to keep the camera balance, ensure that the horizontal state of the camera. It is convenient to adjust the camera. Advantages: simple structure, flexible lifting, a wide range of observation. Disadvantages: the structure occupies slightly larger space, and a camera balance joint [1].

\section{Scheme design}

The network communications and image transmission is fully integrated in a ARM architecture, the architecture of A9 quad core processor based on UBUNTU operating system, at the same time in the embedded system to open a TCP to serial channel. Because most of the module interface is not exactly the same, only part of the module supports analog signals, some modules also have a completely different protocol instructions. To achieve the following module replacement but does not affect the main structure of software, the controller must be completely achieve control commands through the TCP serial transmission channel, protocol of data exchange between each module and controller is convenient. 


\subsection{Harmful gas detection}

The display panel unit adopts industrial screen, connected to the main controller online through the serial port. Can also use HDMI to directly connect to raspberry pie, HDMI screen directly displays in the raspberry pie system inside, reducing the pressure of MCU.

Despite the WIFI communication distance is about 1000 meters, but because of the inherent properties of microwave, it also determines the distance only high in the open environment, in the narrow tunnel environment in general communication distance is less than 200 meters, so it is need to use high power router, antenna and multi AP network structure. According to the antenna installation requirements, select high power and high gain antenna panel directional antenna and microwave antenna disc. Router with higher power value, therefore we chose the $842 \mathrm{w}$ router, as shown in figure 4.

The robot move in the curved tunnel, the body must adopt WIFI mode to transmit information to the control box. The position of control box is fixed relative to the network, and it is equipped with industrial control computer, so it can be used in small power WIFI network card and network connection [2]. the establishment of control network and WIFI box body in motion is the most important control link, so we adopt high power directional antenna (AP or router way). Small blind area, AP or less router advantage, and number of interrupt delay time and antenna are within acceptable limits. The communication structure as shown in figure 5.

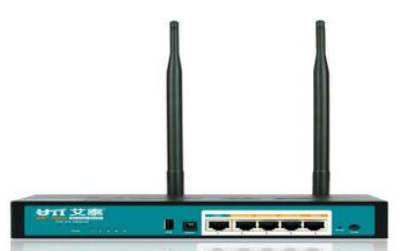

Fig.4 router

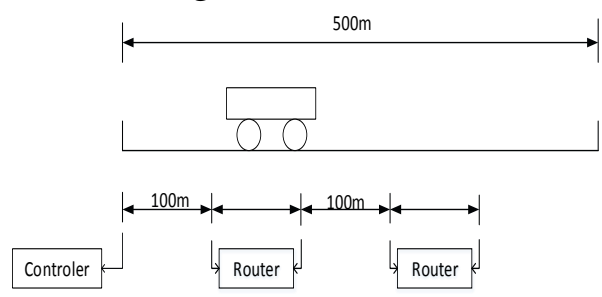

Figure 5. Communication structure.

Above all knowable process of robot cruise, the robot can receive main commands from a remote controller through WIFI, and can judge for themselves to avoid obstacles and barriers, and check the screen cable information, the related message can be transmitted to the remote control box through WIFI. It is convenient for the operators to check the cable line, and don't worry too much about the operation problem of the robot itself and greatly improve the inspection efficiency.

\section{Conclusion}

The robot control scheme is a combined control scheme of three high mature, practical and cost-effective modules. ARM processor for TCP signal processing, and the WIFI is a high frequency radio signal, just using the raspberry pie signal conversion between the two, so as to solve the problem of communication and control of cable tunnel in narrow environment. But for the WIFI module automatically switched AP network, there is no very good solution, only the use of the robot in the tunnel within a short period of time to move, and to make AP switch. There is a brief dropping state may occur while switching AP. Therefore, future research will focus on a better solution to realise stable control in the environment within the narrow tunnel.

\section{References}

[1] Zhou Fengyu, Li Yibin, Wu Aiguo, et al. Design and implementation of [J], mechanical science and technology, 25 (5), (2006).

[2] Zhang Yunchu, Liang Zize, Tan Min, [J], overhead power line inspection robot in robot, 26 (5) (2004). 